\title{
CULTURA E IDENTIDAD. Discusiones teóricas-epistemológicas para la comprensión de la contemporaneidad
}

\author{
Patricia Jimena RIVERO; Virginia Soledad MARTÍNEZ \\ CONICET, Universidad Nacional de Córdoba (Argentina) \\ patriciaj.rivero@gmail.com, mumymartinez@hotmail.com
}

\begin{abstract}
CULTURE AND IDENTITY. A theoretical and epistemological analysis for the understanding of contemporaneity
\end{abstract}

Resumen: En este artículo se presentan algunas reflexiones sobre los conceptos cultura e identidad que durante mucho tiempo suscitaron fuertes debates en el campo de los estudios sobre la cultura y los estudios culturales. Estos términos han sido usados teórica y políticamente como sinónimos intercambiables, lo que ha desembocado en la necesidad de revisar las diferentes perspectivas teóricas usadas hasta ahora. Desde el esencialismo más radical, hasta las posturas enteramente subjetivistas, se desarrolló una discusión de negación mutua que vino a estancar los debates y sus enfoques resultaron, en cierta forma, insuficientes para abordar dichos conceptos. Con la finalidad de comprender la complejidad del mundo contemporáneo, se plantean los marcos interpretativos de los conceptos en articulación con nociones como poder, desigualdad, heterogeneidad, historicidad y conflicto, las cuales con su llegada, vendrían a plantear nuevas discusiones que hasta entonces habían quedado desplazadas. De esta forma, la propuesta de este trabajo es ordenar el complejo mapa de perspectivas conceptuales a través de los aportes de diferentes autores. En este marco, se destaca, el esquema teórico formulado por Alejandro Grimson en Los límites de la Cultura, el cual plantea nuevas conceptualizaciones para dar cuenta de los entramados sociales complejos del mundo contemporáneo.

Abstract: This article presents some considerations about the concepts of Culture and Identity which have historically aroused a heated debate in the studies of culture and cultural studies field. From the theoretical and political perspectives, both terms have been used interchangeably. This created the need of reviewing the various theoretical perspectives currently used. A discussion of mutual negation developed among those who support the most radical essencialism and those who adopt a subjetivism-based position, bringing the debate to a standstill. As a result, the different approaches were not solid enough to address such concepts. In order to understand the complex contemporary world, the interpretation frames of the concepts are associated with the ideas of power, inequality, heterogenity, historicity and conflict, which generates new discussions that had been so far disregarded. Therefore, this piece of work is intended to organize the complex range of perspectives related to this concepts by considering different insights and contributions from several authors. One of the most remarkable theoretical diagrams is the one provided by Alejandro Grimson in Los Límites de la Cultura (The Limits of Culture) in which the author establishes new conceptualizations to account for the complex social structures of the contemporary world.

Cultura. Identidad. Configuración cultural. Intersubjetivismo configuracional. Objetivismo.

Palabras clave: Subjetivismo. Epistemología. Contemporaneidad

Culture. Identity. Otherness. Cultural configuration. Configurational inter-subjectivism. Objectivism. Subjectivism. Epistemology. Contemporaneity 


\section{Introducción ${ }^{1}$}

Desde la década de 1960 se viene dando un intenso debate dentro del campo de los estudios sobre la cultura y los estudios culturales ${ }^{2}$ en torno a los conceptos de cultura e identidad. Lo más sobresaliente de este debate conceptual han sido sus usos ético-políticos, los cuales tuvieron fuertes consecuencias epistemológicas y metodológicas (Grimson, 2008). Así, desde el esencialismo más radical, hasta las posturas enteramente subjetivistas, se desarrolló una discusión de negación mutua que vino a estancar los debates y sus enfoques resultaron, en cierta forma, insuficientes para abordar dichos conceptos. Desde los estudios culturales varios autores se han tomado el trabajo de revisar estos enfoques (García Canclini, 1999, 2001; Martín-Barbero, 1987; Grimson, 2003, 2007, 2010, 2011; Hall, 1996; Williams, 1980) con la idea de promover un esquema teórico con aplicabilidad a los contextos híbridos e interculturales actuales caracterizados por la intensa interconexión global. Es innegable que la cultura y la identidad se presentan como términos necesarios para comprender los mundos contemporáneos, y una distinción precisa entre ambas nociones resulta imprescindible para el análisis de los procesos sociales.

El objetivo de este artículo es presentar distintas perspectivas sobre el uso conceptual de cultura e identidad, reflexionando acerca de los modos en que operan en ellas las nociones de poder, desigualdad, heterogeneidad, historicidad y conflicto. Para ello, trazamos un recorrido por bibliografía especializada en el campo de estudios sobre la cultura y los estudios culturales, tomando los aportes de diferentes autores que problematizan los alcances, las limitaciones y las implicaciones políticas de los distintos usos de dichos conceptos.

A modo de organización y articulación de las diferentes perspectivas teóricas, retomamos el esquema propuesto por Alejandro Grimson en Los límites de la Cultura, en el que da cuenta de "tres posiciones lógicas de la teoría social" (2011, p.18): el objetivismo, el subjetivismo y la intersubjetividad configuracional, en una mirada posconstructivista que habilita avanzar en el conocimiento de la relación entre cultura, identidades y política.

La organización expositiva de este artículo consta de dos grandes apartados dentro de los cuales se encuentran tres secciones que abordan la perspectiva objetivista, la subjetivista y la del intersubjetivismo configuracional. En el primer apartado, pondremos en discusión los enfoques teóricos sobre el concepto de cultura, mientras que en el segundo abordaremos el debate en torno al concepto de identidad. Advertimos que, como esquema, no sugerimos una clasificación perfectamente delimitada de la teoría social ni proponemos inscribir a cada autor en una única perspectiva, sino que se trata de identificar aquellos elementos teóricos, epistemológicos y políticos que los acercan a una u otra posición.

\footnotetext{
$1 \mathrm{El}$ presente trabajo recoge reflexiones personales surgidas a partir de lecturas y discusiones en el marco del seminario "Procesos culturales y comunicaciones en América Latina" del Doctorado "Estudios Sociales de América Latina" del Centro de Estudios Avanzados de la Universidad Nacional de Córdoba (CEA-UNC), a cargo del Dr. Alejandro Grimson y del Dr. Sergio Caggiano. Agradecemos a Nicolás Cabrera (IDAES/UNSAMCONICET), a Gonzalo Assusa (CIFFYH-CONICET) y Carlos Alberto González Zepeda (UAM-CuajimalpaCONACYT) por los comentarios a la versión preliminar de este trabajo.

2 Siguiendo a Eduardo Restrepo, "hay que distinguir entre estudios culturales y estudios sobre la cultura porque los estudios culturales constituyen un proyecto intelectual y político que: 1) concibe la cultura-como-poder y el poder-como-cultural; 2) suponen un enfoque no reduccionista que se expresa en una actitud transdisciplinaria; 3) implican una vocación política que busca intervenir sobre el mundo; y 4) su encuadre es el contextualismo radical (con respecto a su forma de teorización, a las metodologías utilizadas, a su conceptualización de la política y su propio proyecto)" (2015, p.7). Por su parte, concibe los estudios sobre la cultura como abordajes dentro de los cuales prima la cultura o lo cultural como categoría central de análisis. La antropología cultural, la sociología de la cultura, la crítica cultural o la historia cultural son algunos de los enfoques más importantes de los estudios sobre la cultura.
} 


\section{Produciendo cultura: entes, ficciones y configuraciones}

Un antecedente histórico importante para situar el terreno fértil dentro del cual reemerge la discusión en torno al concepto de "cultura" es, sin lugar a dudas, la finalización de la segunda guerra mundial donde el Holocausto terminó deslegitimando todos los argumentos racialistas desacreditando cualquier criterio biológico como sistema clasificador de la sociedad (Grimson, 2008). Los principios del racismo por parte del Estado alemán comenzaron a desdibujarse y de manera sorprendente "la cultura pasó a desempeñar la función de categoría clasificatoria que la raza ya no podía cumplir, y cobró legitimidad como argumento" (Grimson, 2011, p.62). Es en este contexto -y con un claro posicionamiento político- que se abandona el uso de "raza" a favor del concepto "cultura". Aunque las intenciones eran buenas, esta nueva mirada también implicó nuevos problemas, porque se sustituyó la imagen de un mundo dividido en razas por la de un mundo dividido en culturas o áreas culturales (Grimson, 2008; 2011).

La concepción de cultura surge así desde un marco puramente objetivista donde los hechos sociales son cosas, materia definida y organizada, y el investigador es apenas un instrumento de análisis que observa cómo funciona tal realidad. En efecto, la denominación "objetivismo" alude a la ausencia de valoraciones subjetivas del sujeto que investiga, y, por ello, a su posibilidad de desplegar una cientificidad dura que le permita alcanzar la validez de sus proposiciones. Algunas de las variantes de este tipo de abordaje son el naturalismo, el realismo clásico, el positivismo, el estructuralismo clásico, el esencialismo y el funcionalismo (Grimson, 2011).

Mediante este abordaje, se diseñaron propuestas para el análisis cultural que tienden a la homogeneización de los sujetos y a su división esquemática en grupos con ciertas características compartidas, fronteras más o menos claras y una identidad propia. Así, los antropólogos observan la identidad como una cuestión subsidiara y definida desde la cultura. Grimson apela a la metáfora de "archipiélago" $(2011$, p.58) para referir a esta forma de comprender las culturas como espacios-tiempos cerrados y homogéneos al interior de sus fronteras. El programa antropológico es, en ese marco, conocer y conservar esta diversidad cultural. En esta línea, algunos postulados de Samuel Huntington son muy elocuentes. Según el autor, el concepto de cultura,

"hace referencia a la lengua, a las creencias religiosas, a los valores sociales y políticos, a las concepciones de lo que está bien y lo que está mal, de lo apropiado y lo inapropiado, y a las instituciones objetivas y las pautas de comportamiento que reflejan esos elementos subjetivos" (2004b, p.31, la traducción es nuestra)

Un elemento clave en el pensamiento de Huntington es el concepto de civilización, que lo define como un "agrupamiento cultural" (2004, p.48). Aldeas, ciudades, regiones, grupos étnicos, nacionalidades y grupos religiosos son, para el autor, todas culturas distintas con diferentes niveles de heterogeneidad cultural. Una civilización es, de esta manera, la más elevada agrupación cultural de personas y el más amplio nivel de identidad cultural que poseen los pueblos (Huntington, 2004). Se define tanto por elementos objetivos comunes (idioma, historia, religión, costumbres, instituciones), como por autoidentificación subjetiva de los sujetos.

El autor propone "ver el mundo desde la perspectiva de siete u ocho civilizaciones, [cuya] distinción principal es la que se hace entre Occidente como civilización dominante hasta ahora y todas las demás" (Huntington, 2004, p.39, la cursiva es nuestra). La expresión todas las demás conlleva el intento de abandonar el pensamiento dual Occidente-Oriente 
objetado por varios autores 3 ; intento que a nuestro entender es fallido ya que cae en otra dualidad: Occidente-el resto del mundo. Sobre ello, Huntington argumenta que la fertilidad de su proposición radica en que "al menos implica la existencia de muchos no-Occidentes" (2004, p. 35) que tienen poco o nada en común entre ellos. De esta forma, en este mapamundi civilizacional, la heterogeneidad parece encontrar lugar entre las distintas civilizaciones, pero poco se dice acerca de cómo trabaja esa diferencia hacia adentro de ellas, especialmente, de la civilización occidental cuya diversidad nunca es problematizada por el autor.

Las implicancias políticas de esta manera particular de no plantearse preguntas sobre el lugar desde el cual procede el propio pensamiento no son menores, ya que hace suponer que proviene de ningún sitio o, lo que es igual, de todos los sitios, asumiendo así que ese conocimiento es generalizable y universalizable (Chakrabarty, 2008). Esto nos acerca a las discusiones en torno a la colonialidad del saber ${ }^{A}$ como herramienta de dominación que buena parte de los autores inscriptos en las corrientes poscoloniales y decoloniales vienen denunciando ${ }^{5}$. Más adelante, abordaremos la noción de situacionalidad radical de Grimson que cristaliza, a nuestra entender, algunas de estas discusiones.

Otro aspecto característico de estas perspectivas culturalistas clásicas es pensar a las sociedades en esferas, dentro de las cuales la cultura es una de ellas. Tomemos como ejemplo el planteamiento de Clifford Geertz (autodenominado funcionalista aunque con ciertos reparos) $)^{6}$ : el antropólogo estadounidense propone que "los elementos dinámicos del cambio social [...] surgen de la circunstancia de que los esquemas culturales no s[o]n completamente congruentes con las formas de organización social" (1987, p.132, las cursivas son nuestras).

Ante la distinción que hace Geertz entre los esquemas culturales por un lado y las formas de organización social por el otro, es interesante retomar la crítica de Raymond Williams (1980) a la clásica definición marxista de estructura y superestructura como áreas o elementos separados y, especialmente, a su compleja relación de determinación. En este sentido, Williams afirma que no existen esferas y que, como tal, la cultura no es una esfera separada de (ni determinada por) otras, como puede ser la economía o la política. Por el contrario, la define como un proceso social total en el que las personas definen y configu-

\footnotetext{
3 Edward Said, por ejemplo, realiza una aguda crítica a lo que denominó orientalismo, en tanto "sistema de pensamiento [que] se acerca a una realidad humana heterogénea, dinámica y compleja desde una postura esencialista y no critica, lo que sugiere la existencia de una realidad oriental perdurable y de una esencia occidental opuesta, pero no menos perdurable, que observa Oriente desde lejos y, ipor qué no decirlo!, desde arriba" (2004, p.438)

4 La colonialidad del saber "se refiere al efecto de subalternización, folclorización o invisibilización de una multiplicidad de conocimientos que no responden a las modalidades de producción de 'conocimiento occidental' asociadas a la ciencia convencional y al discurso experto. [...] La pretensión de universalidad, objetividad y neutralidad del 'conocimiento occidental' es donde se afinca su supuesta superioridad epistémica que inferioriza o invisibiliza las formas de concebir y producir conocimientos diferentes. [...] El distanciamiento de sí, la no situacionalidad, con la pretensión de producir un conocimiento de alcance universal desde un punto de vista que subsume todos los puntos de vista pero que no aparece como tal, es llamada por Castro-Gómez la hybris del punto cero" (Restrepo, 2010, p.136-138)

5 Eduardo Gruner realiza un recorrido por los fundamentos teórico-epistemológicos de la teoría poscolonial y la teoría del sistema-mundo capitalista para luego ensayar su aplicación a una problemática específica: la relación entre ciertas formas de la literatura y la expresión estética y la (re)construcción de "identidades" colectivas (étnico-nacionales) en el contexto de la mundialización capitalista poscolonial. (2002, pp. 167-249).

6 Geertz reconoce que el funcionalismo presenta ciertos déficits para explicar el cambio social ya que, desde un enfoque estático y ahistórico, prevalecen los aspectos funcionales, de armonización y de integración de los usos y costumbres sociales antes que los disfuncionales, desorganizadores y desintegradores. Dice, entonces, que una forma de teoría funcional más dinámica, que reconozca la tensión entre la necesidad de las personas de hacer sentido del mundo en el que viven con la necesidad de hacer funcionar el organismo social, sería capaz de explicar mejor los cambios sociales.
} 
ran sus vidas. Retomaremos más adelante otros postulados de Williams, especialmente su noción de hegemonía ${ }^{7}$.

A partir de las décadas de los 70 y 80, el enfoque objetivista fue fuertemente cuestionado. Los procesos migratorios (no tanto producto del incremento cuantitativo como del desplazamiento de las poblaciones antiguamente colonizadas hacia los Estados Unidos y Europa), junto con los cambios tecnológicos y el papel de las nuevas redes comunicacionales, volvió inviable la interpretación de otras culturas como si fueran mundos distantes (Beck, 2002; Hannerz, 2003; Grimson 2010, 2011). Así, la imagen de un mundo dividido en culturas armónicas y estables se tornó inverosímil y se evidenciaron las consecuencias teóricas y políticas que implica desconocer la interconexión (desigual) entre los grupos humanos así como sus heterogeneidades, conflictos y desigualdades internas.

Frente a la idea de las culturas como cosas, el subjetivismo (en sus variantes constructivistas, deconstructivistas y posmodernistas) muestra, en sus posiciones más extremas, que son ficciones del investigador y que "lo real" sólo existe como percepción o idea. Reivindicando la agencia frente a la estructura, propone a un individuo libre de sujeciones y concepciones más fragmentarias de lo social. En el plano cultural, cuestiona fuertemente la idea de archipiélagos (es decir, la existencia de diferentes culturas como si fueran mundos distantes), enfatiza el carácter borroso de las fronteras e híbrido de las culturas y, en sus posiciones más radicales, cuestiona cualquier acepción del concepto de cultura ya que todas ellas producen alteridades y fabrican fronteras, más allá del contexto teórico y político en el que se utilizan.

Lila Abu-Lughod (2005, 2012 [1991]) y Edward Said (2013) realizan fuertes críticas al uso del concepto de cultura. En sus planteos, establecen que la noción de cultura encarcela a las personas no occidentales dentro de límites establecidos por la homogeneización, negando el tiempo y el conflicto de lo que está dentro de esos límites y haciendo de ello algo fijo y esencial. Así, la cultura opera en el discurso antropológico como una herramienta para hacer al otro y a las diferencias, instalando distinciones que no sólo pretenden ser naturales sino que conllevan una jerarquía implícita. Si bien la diferencia no tiene porqué ser axiológica, la jerarquización, muchas veces derivada de la posición, se entremezcla inadvertida en ella.

Es por esto que Abu-Lughod insta a los antropólogos a desarrollar estrategias para escribir contra la cultura, siendo su propuesta metodológica y epistemológica la de hacer "etnografías de lo particular" (Abu-Lughod 2012 [1991], p.130). Al enfocar en lo particular se subvierten las connotaciones más problemáticas de la cultura: homogeneidad, coherencia y atemporalidad. Subraya la autora que las múltiples interacciones globales en el presente, imposibilitan pensar a comunidades como unidades aisladas y nos imponen analizar la forma en que los efectos de las fuerzas y dinámicas que no son locales se manifiestan "sólo local y específicamente [y] se producen en las acciones de individuos que viven sus vidas de manera particular" (2012, p.148). En su trabajo La interpretación de la(s) cultura(s) después de la televisión (2005), la autora ofrece ejemplos de cómo las mujeres de una aldea egipcia establecen relaciones diferentes con los turistas, periodistas, antropólogos, habitantes de El Cairo cuando viajan a la ciudad y con la televisión que, por su "ubicua presencia

7 Si bien traemos a Williams con su noción de hegemonía, las discusiones en torno a este concepto suelen ubicarse en el trabajo teórico de Antonio Gramsci. En sus Cuadernos de la cárcel, entre otros trabajos, el autor propuso una caja de herramientas conceptuales que permitieran entender las formas históricas concretas en la que ciertos grupos o clases ejercen dominación sobre otros. Se centró específicamente en los mecanismos políticos y culturales que dan sustento a esas formas de dominación. 
en la vida e imaginarios de las personas del mundo contemporáneo" (2005, p.59), parece ser una parte clave de las interconexiones con personas o textos extralocales. Es en este sentido que la autora sostiene que la televisión vuelve cada vez más problemático el concepto de cultura como comunidad localizada de personas que se sostienen en redes compartidas de significación.

Christopher Brumann (1999) discute con el movimiento de escribir contra la cultura, postulando que, a pesar de los usos equivocados del concepto, existe un "uso óptimo" que justifica mantenerlo. Desde una perspectiva distribucional, el autor define a la cultura como "un conjunto de rutinas específicas aprendidas (y/o sus productos materiales y no materiales) que son característicos de un grupo delineado de personas" $(1993, \mathrm{p} .6)$ y que esa delimitación está dada por una distribución no azarosa, aunque no perfecta, de atributos individuales. Brumann entiende que un riesgo importante que conlleva abandonar el uso del concepto de cultura es que parezca que se está indicando que esos atributos se distribuyen al azar.

Con todo, las posturas subjetivistas se volvieron triviales, y en su afán por rebatir al objetivismo se estancaron los debates en una relación determinista entre sujeto y estructura. Esta disputa epistemológica obstaculizó un abordaje serio en torno a la cultura y la identidad. Es en este contexto que nace la crítica al constructivismo, a la invención social y al paradigma posmoderno multiculturalista que, abonando un falso discurso de igualdad social, reconoció la diversidad y el derecho a la diferencia pero esto no incluyó el reconocimiento de las desigualdades dadas a partir de estas diferencias.

La pretensión del multiculturalismo en algunas formulaciones era invertir o modificar la valoración habitual de grupos o colectividades discriminados -como los pueblos originarios, los afro, los inmigrantes excluidos, los gays, las lesbianas y los transexuales, entre muchos otros- y reivindicar, entre sus derechos civiles, su derecho a la diferencia. Gracias al papel de los nuevos movimientos sociales en respuesta a las políticas de discriminación, asimilación y homogenización, las políticas muticulturalistas comenzaron a imponerse en el mundo académico y en el diseño de políticas públicas. No obstante, con esta concepción de lo cultural, se extendió la lógica de la discriminación porque al concebirse la diferencia cultural como algo inmutable, con fronteras fijas que separan ciertos grupos de otros se reforzó la idea del "nosotros" y "ellos", posicionándose cada colectivo en forma de mosaicos culturales. Grimson (2011) apunta a que tanto los que discriminan como quienes pretender reconocer a esos grupos comparten el supuesto de que el mundo está dividido en culturas con identidades cristalizadas.

A pesar de que estas perspectivas subjetivistas realizaron aportes significativos incorporando las nociones de historicidad, poder, subjetividad, construcción y deconstrucción, postularon el fin de conceptos como nación, territorio, frontera o identidad. Así, a partir de las numerosas contribuciones de diversos autores clásicos y contemporáneos, la pregunta teórica y política para el análisis cultural es cómo construir una perspectiva posconstructivista que

"despliegue una crítica a los mundos construidos sobre la base del desprecio a las multiplicidades que los constituyen, y una crítica simultánea a las proyecciones de desintegración y fragmentación que apelan instrumentalmente a las nociones de diversidad, pero sin aludir nunca a los poderes reales que trabajan esas diferencias" (Grimson, 2011, p.50).

En efecto, para el programa posconstructivista la diversidad no debe comprenderse como un mapa esencializado y trascendente de las diferencias sino como un proceso abierto y dinámico, un proceso relacional vinculado a las desigualdades y las relaciones de poder. 
Dicho en otras palabras, la diversidad -en tanto que deviene de una relación humana- es histórica, política, situada, conflictiva y procesual (Grimson, 2011).

El intersubjetivismo configuracional, por su parte, recupera la definición subjetivista de las prácticas sociales como inventos humanos, pero sin negar por ello su carácter de real. Estas prácticas sociales son, entonces, ontológicamente subjetivas (creaciones humanas) y epistemológicamente objetivas (con efectos reales en los modos de percepción, significación y acción) (Grimson, 2011). Lo significativo es que ante la pregunta por la relación sujeto-estructura, mismo que por la relación material-simbólico, se abandona la pretensión de una respuesta trascendente y se asume que es histórica, situada y casuística.

En este marco es que Grimson propone la noción de configuración cultural que, a diferencia del concepto de cultura de Brumann (1999), no se pregunta por los rasgos de los individuos y su distribución, sino que lo hace por los espacios y los regímenes de sentido. Las configuraciones culturales, aludiendo a espacios donde hay tramas simbólicas compartidas, desigualdades de poder sedimentadas e historicidad, enfatizan la heterogeneidad y el hecho de que ésta se encuentra, en cada contexto, articulada de un modo específico. Esta articulación toma sentido en tanto sea observada a la luz de otros entramados también heterogéneos.

Lo interesante, en todo caso, es que la contingencia de esa articulación de diversidades supone también la contingencia de sus fronteras (Grimson, 2011), entendidas como regímenes de significación diferenciados y percibidos por sus propios participantes. En este sentido, las configuraciones culturales buscan articular y superar tanto la tradición culturalista como la posmoderna instrumentalista, con el objetivo de construir respuestas adaptadas a la complejidad del mundo contemporáneo. De esta forma, la noción de configuración cultural de Grimson se solidariza con el planteo de Abu-Lughod (2005) que presentáramos párrafos atrás, en la medida en que la aldea egipcia que la autora analiza, y las diferentes formas de interconexión que en ella se dan, constituirían una configuración cultural, en tanto los conflictos y relaciones que allí emergen nada tienen de aleatorios, sino que existe una trama simbólica común donde esas diferencias son mutuamente comprendidas.

En toda configuración cultural, en tanto espacio de relaciones sociales, existe circulación de poder. Williams (1980) introduce en este punto del análisis cultural la noción de hegemonía. La cultura como proceso social total está siempre atravesada por distribuciones específicas de poder y relaciones de dominación-subordinación que configuran procesos hegemónicos que son relacionales, históricamente situados y dinámicos. Vale la pena aclarar que la hegemonía nunca es absoluta, sino que supone la existencia de procesos contrahegemónicos, más o menos confrontativos, más o menos autónomos que, enfrentando o desconociendo las propuestas hegemónicas, operan allí donde la hegemonía instituye un sentido del ridículo para la acción, ampliando las fronteras de lo posible y es justamente en ese punto donde radica su potencial instituyente (Williams, 1980; Grimson, 2011; Caggiano, 2012).

Quisiéramos, finalmente, volver sobre un asunto que insistimos en párrafos anteriores: la historicidad en el análisis cultural, es decir, su inexorable relación con un tiempo y un lugar específico o, en palabras de Grimson, su situacionalidad radical (2011, p. 36). Esta idea nos invita a reflexionar acerca de la "relación necesariamente inestable entre toda idea abstracta y su instanciación concreta" (Chakrabarty, 2008: 19). Objetando la idea de la universalización del conocimiento, Grimson plantea la necesidad no tanto de abandonar formulaciones abstractas sino de atender al modo específico que aquellas asumen en una situación determinada.

A modo de cierre de este apartado y para introducirnos a la discusión que proponemos en la segunda parte del trabajo, traeremos a colación el concepto de "frontera" como no- 
ción clave para el análisis cultural y diremos que las fronteras culturales son fronteras de significación y las identitarias son fronteras de sentimientos de pertenencia, y que rara vez coinciden unas con otras (Grimson, 2011).

\section{Identidad: entre esencialismos y constructivismos}

La falta de una distinción clara entre identidad y cultura ha generado un problema teórico dentro de la antropología en la que se entremezclan rutinas cotidianas, creencias y rituales con los sentimientos de pertenencia y su intensidad. De allí la idea que si en un momento hay menor intensidad o difuminación de sentimientos nacionales eso implica que se desdibuja la cultura. Y viceversa: el esencialismo postula que toda apropiación e hibridación cultural es una pérdida de identidad. Así, es realmente un problema que se los considere sinónimos o automáticamente interdependientes (Grimson, 2010: 3).

En las vertientes esencialistas, las identidades aparecen como un reflejo de un listado de rasgos culturales objetivos compartidos o bien como una expectativa que busca explicar lo que la gente hace o debería hacer en base a quiénes son o a qué cultura pertenecen. Son enfoques que postulan un sentido de determinación o correspondencia necesaria entre pertenencia y comportamiento: es decir, las identidades son prescriptivas de una manera de comportarse (Briones, 2007: 60). Esta idea de vínculo de implicación simple entre cultura e identidad o, dicho de otra forma, de superposición de las fronteras culturales e identitarias (Grimson, 2011), refuerza la proposición fundamental del esencialismo cultural: las identidades derivan de la cultura. Brubaker y Cooper (2001) llaman conceptos fuertes o duros a estas formas de definir la identidad. Estos suponen, en el plano colectivo, una igualdad fundamental entre los miembros de un grupo que va a manifestarse como una solidaridad con los otros miembros, es decir, como una acción colectiva. Implican, entonces, nociones fuertes de límite y homogeneidad entre los miembros y, al mismo tiempo, una marcada distinción de los no miembros, lo que determina un límite claro entre el adentro y el afuera. En el plano individual, la identidad aparece como aspecto central de la conciencia del ser individual, evocando algo profundo y perdurable a ser preservado más que algo superficial y efímero. En palabras de Stuart Hall (1996), son enfoques que postulan la idea de núcleo estable del yo, como así también la de un yo verdadero que se oculta bajo los muchos otros yos superficiales y artificialmente impuestos, que un pueblo con una historia y una ascendencia compartida tiene en común.

Así, estos conceptos fuertes, al evocar una igualdad fundamental, profunda y perdurable, dan lugar a un doble fenómeno: primero, como mencionamos en el primer apartado al referirnos a los cuestionamientos que Abu-Lughod (2012 [1991]) y Said (2013 [1978]) realizan al concepto de cultura, se invisibiliza el conflicto y las diferencias hacia el interior de fronteras identitarias aparentemente fijas y esenciales que separan mundos homogéneos en su interior, y de esta forma se puede estar legitimando las relaciones de desigualdad que allí operan. Segundo, se concibe al otro esencialmente diferente, distante e inconmensurable. Traemos aquí la noción de racismo como expresión extrema para ejemplificar la construcción de la alteridad bajo estos criterios. Este presupone agrupamientos humanos cuyos miembros poseen características físicas comunes, la división del mundo en razas, la continuidad entre lo físico y lo moral, la determinación de las diferencias culturales por las físicas, la transmisión hereditaria de lo mental, una jerarquía única de los valores (Todorov, 1991). Este último aspecto es clave para entender cómo juega el poder y el conocimiento en las relaciones que se establecen bajo estas presuposiciones: hay razas superiores y razas inferiores, rechazables. En efecto, "cualquier tentativa de encasillar a culturas y pueblos en castas y/o en esencias separadas y diferentes está expuesto no solo a los equívocos y las falsedades consiguientes, sino también a que nuestra comprensión se alíe con el poder" (Said, 2013 [1978]: 456). La naturalización de las fronteras identitarias constituye lo que 
Grimson denominó "el fetichismo de las identidades" (2011: 26), que ocluye las prácticas y las condiciones sociales que las convirtieron en objetos, es decir, descontextualiza, deshistoriza el análisis.

El constructivismo viene a poner en cuestión el esencialismo apuntando al carácter borroso de las fronteras e híbrido de las culturas. Los relatos nacionales que referían a la homogeneidad fueron desacreditados, no sólo por procesos de globalización, sino por dinámicas emergentes indígenas, afro, mestizas y regionales desde abajo que repusieron la distancia entre territorio jurídico, la cultura en el sentido tradicional y las identidades (Grimson, 2010:4).

Claudia Briones (2007) planeta que Frederik Barth ${ }^{8}$ es un antecesor en el esfuerzo por pensar las identidades de maneras que se distanciaran de posiciones esencialistas. La autora postula que fue a partir de él que se empezó a pensar las identidades como inevitablemente contrastivas, socialmente construidas y cambiantes en sus contenidos, deviniendo estas ideas con el tiempo en certezas o sentido común en las investigaciones. Pero este reconocimiento trae aparejado otra certeza (problemática a los ojos de Briones) de presuponer que todo límite opera relacional pero contrastivamente en base a la estricta y duradera separación ellos-nosotros, de allí que trabajos deconstructivistas posteriores problematicen esta idea de contraste. Briones va a discutir la idea de contrastividad señalando que "en tanto sistema de representaciones anclado en la codificación de diferencias, las identidades sociales pueden (y no deben) presentarse como contrastivas" (2007: 74).

Para ordenar las perspectivas que conforman al enfoque constructivista nos valdremos del esquema que propone Briones (2007), en el que presenta movimientos más bien paralelos que sucesivos. En primer lugar, autores como Edward Said (2013 [1990]), Benedict Anderson (1991), y Howsband y Ranger (1989) que, acentuando la desnaturalización y la historización de ciertas ideas, cuestionaron aquella de identidad como causa de un estado de cosas. En segundo lugar, enfoques deconstructivistas que cuestionaron ciertos conceptos claves sin proponer una superación ni tampoco abandonar su uso, sino que postulan seguir usándolos pero afuera del paradigma bajo el cual se originaron.

Stuart Hall (1996) es quien representa, en el esquema de Briones, el segundo movimiento, aunque alguna de sus ideas se inscriban también en lo que al comienzo de este trabajo denominamos (siguiendo a Grimson) intersubjetivismo configuracional. Ni deconstructivista ni constructivista radical, el autor sugiere que la identidad es "una idea que no puede pensarse a la vieja usanza, pero sin la cual ciertas cuestiones claves no pueden pensarse en absoluto" (1996, p.14). Pensando en la correspondencia (o no) entre posiciones, condiciones, dispositivos o reglas (a lo que Briones siguiendo a Foucault denominó sujeción) y la manera de instalarse, ocupar, ser regulado o atravesado por ellas (subjetivación), Hall incorpora la idea de correspondencia no necesaria, distanciándose tanto del esencialismo -que ve a las identidades como fijas y naturales por partir de una idea de correspondencia necesaria- y del antiesencialismo -que, al partir de una necesaria no correspondencia postula ideas relativas y volátiles de identidad donde las elecciones dependen de la voluntad de los individuos-.

Esta idea lleva a Hall a entender cualquier identidad como efecto de un trabajo de articulación y como punto de sutura emergente de procesos de identificación. El concepto de articulación acepta que las identidades nunca se unifican y, en los tiempos de la modernidad tardía, están cada vez más fragmentadas y fracturadas; nunca son singulares, sino construidas de múltiples maneras a través de discursos, prácticas y posiciones diferentes, a menudo cruzados y antagónicos. Están sujetas a una historización radical, y en constante proceso de cambio y transformación (Hall, 1996, p.17). Las identidades, en consecuencia, se cons-

8 Antropólogo social noruego, cuya obra "Los grupos étnicos y sus fronteras" (1969) representó un gran aporte para pensar las identidades étnicas. 
tituyen dentro de la representación y no fuera de ella. Se relacionan tanto con la invención de la tradición como con la tradición misma, y nos obligan a leerla no como una reiteración incesante sino como "lo mismo que cambia" (Gilroy 1994, citado en Hall 1996).

Retomando la idea de Brubaker y Cooper (2001) de conceptos fuertes o duros de la identidad que planteamos anteriormente, los autores postulan en contraposición a los denominados conceptos débiles o blandos de definir la identidad, inscriptos en enfoques constructivistas. Indican, así, distintos usos blandos: aquellos que entienden a la identidad tanto como base para la acción social y política, como así también producto de la misma; aquellos que la ven como el producto de discursos múltiples, iluminando la naturaleza inestable y fragmentaria del "yo"; aquellos que postulan que está determinada por la posición en el espacio social, pero no una posición en una estructura social universalísticamente concebida, sino a una posición en un espacio multidimensional definido por atributos particulares. Los autores cuestionan la propuesta de Hall de seguir utilizando el concepto de identidad a pesar de las ambigüedades que representa, y proponen, en cambio, reemplazarlo por otros términos (identificación, categorización, autocomprensión, locación social, comunidad, conexionismo, grupalidad) que expliquen mejor a qué se hace referencia cuando se habla de identidad.

Es así, pues, que bajo la crítica de los conceptos blandos (débiles) y duros (fuertes), Brubaker y Cooper (2001) centran su atención al uso de identidad como concepto analítico y en sus discusiones se preguntan cuál es la supuesta utilidad del concepto. Señalan que el término es inapropiado para realizar esta labor porque está atravesado por la ambigüedad, dividido por significados contradictorios, y sobrecargado de connotaciones reificadas (Brubaker y Cooper, 2001:58). Asimismo, sostienen que criticar el uso de identidad en el análisis social no es cegarse a la particularidad. Es, en cambio, concebir los reclamos y posibilidades que surgen de afinidades y afiliaciones particulares, de comunalidades y conexiones específicas, de historias y autocomprensiones específicas, de problemas singulares y predicamentos de manera más diferenciada. La idea no es "sensibilizarse" a la particularidad -como lo ha hecho el análisis social en la últimas décadas- sino "ir más allá de la identidad" para lograr una claridad conceptual que permita una mejor comprensión política y un serio análisis de lo social.

En el afán constructivista de desustancializar las identidades se las ha desgajado de aquellas condiciones no esenciales pero sí sedimentadas que atraviesan la acción social, de aquellas

"maquinarias estratificadoras que nos dan acceso diferencial a la experiencia y el conocimiento, [...] que codifican y buscan estabilizar las identidades dentro de un sistema de diferencias autorizadas, y [...] que definen dispares movilidades estructuradas que indican por qué lugares cada cual puede o no moverse, a cuáles cada cual puede o no acceder" (Briones, 2007: 69).

De allí que el énfasis en lo político, la conflictividad y los intereses chocó con los límites de su antiesencialismo (Grimson, 2011), desconociendo las relaciones de poder y desigualdad que median los procesos de construcción de identidades. A este respecto, un elemento clave para la comprensión de estos procesos es la noción de "configuración cultural" para poder comprender procesos en los que una persona puede tener sentimientos de pertenencia (identificación) con otra que vive en una región distante del mundo, y sin embargo sentirse muy lejos -un extranjero- al interior de una comunidad vecina, con quien forma parte del mismo Estado. Esta situación de dualidad sólo se hace visible cuando no se piensa en términos esencialistas, sino cuando se comprende que "la esfera territorial no determina mecánicamente las identificaciones” (Grimson, 2011: 136). En la misma línea, Hannerz señala que "a medida que las personas se desplazan con sus significados, y a medida que 
los significados encuentran formas de desplazarse aunque las personas no se muevan, los territorios ya no pueden ser realmente contenedores de una cultura" (1996: 24).

Trabajando en la construcción de un enfoque posconstructivista, e íntima y complejamente vinculado al concepto de configuración cultural, Grimson propone referirnos a las identificaciones, tomando como punto de partida aquella distinción que hiciéramos anteriormente entre las fronteras culturales y las identitarias, que conlleva el supuesto de que porque algunas personas se sientan pertenecientes a un mismo grupo social no quiere decir que haya homogeneidad cultural. Al referirse a lo cultural, el autor alude a las prácticas, creencias y significados rutinarios y fuertemente sedimentados, mientras que con lo identitario se refiere a los sentimientos de pertenencia a un colectivo y a los agrupamientos fundados en intereses compartidos. La noción de identificaciones tiene una doble implicancia: por un lado remarca el carácter procesual y dinámico de su construcción y definición y, por el otro, reconoce la posibilidad de ser múltiples y simultáneas, es decir, sentirse identificado al mismo tiempo con un club, un barrio, un líder político, etc.

Mientras algunos enfoques postulan que compartir atributos o relaciones implica compartir una identidad, otros autores (Grimson, 2011; Brubaker y Cooper, 2001) sostienen que no hay vínculo de implicación simple entre los aspectos ligados a los atributos sociales y a las relaciones entre las personas con sus sentimientos de pertenencia y sus intereses, y es justamente por ello que la identificación es más una definición de los mismos actores sociales que el resultado de una definición del investigador. Basta mencionar como ejemplo la noción de "comunidad imaginada" de Benedict Anderson, donde los miembros de una nación, a pesar de no tener relaciones sociales con todos los otros miembros, comparten un sentimiento de pertenencia a esa nación. Tampoco existe una correlación necesaria entre los intereses, en este caso referidos a una construcción o articulación de los actores sociales, y los sentimientos de pertenencia. Negar entonces implicaciones simples nos invita a pensar el proceso de construcción de identificaciones en un contexto histórico determinado y en el marco de configuraciones culturales específicas, con sus relaciones de poder y desigualdad. Bien señaló Briones que historizar las identidades, más que dar cuenta de su condiciones de "construidas", implica comprender las disputas y tensiones que las atraviesan. Es allí donde "cada sociedad tiene una caja de herramientas identitarias, un conjunto de clasificaciones disponibles que permiten a sus miembros identificarse a sí mismos e identificar a los otros" (Grimson, 2011: 184).

Dijimos entonces que las categorías identitarias no sólo refieren a los sentimientos de pertenencia de unos sino que también a sus interlocutores, sus otros. Grimson introduce en este punto la noción de interpelación:

"para aludir a los modos en que una persona o grupo o institución se refiere a sus alteridades. Utilizando esa caja de herramientas aludida anteriormente, situada y contextualizada, un miembro de una sociedad se identifica, es interpelado e interpela a los otros [...] En ese proceso de circulación social de categorías y clasificaciones humanas se disputan sentidos, desigualdades, jerarquías y poder" (Grimson, 2011: 186).

En síntesis, para Grimson (2010, 2011), cultura e identidad aluden a aspectos analíticamente diferenciables de los procesos sociales. La relación entre esos dos aspectos no puede presuponerse y generalizarse para todos los casos. Podremos encontrar casos en los cuales en un grupo crecen o decrecen juntas las fronteras culturales y las fronteras identidades, así como podremos encontrar todas las combinaciones posibles entre ambos términos. Lo que resulta indispensable es analizar por separado los aspectos de la cultura y los de la identidad, así como asumir que las respuestas sólo se encuentran en cada caso empírico. Las identidades son, por así decirlo, las posiciones que el sujeto está obligado a tomar, a la vez 
que siempre "sabe" que son representaciones, que la representación siempre se construye a través de una "falta", una división, desde el lugar del Otro, y por eso nunca puede ser adecuada -idéntica- a los procesos subjetivos investidos en ellas.

\section{Reflexiones finales}

A lo largo de este artículo hemos abordado las principales perspectivas que jugaron un papel central en el debate teórico-epistemológico sobre la cultura y la identidad. De la mano de los estudios sobre la cultura, así como de los estudios culturales, nos preguntamos de qué manera es posible construir una perspectiva posconstructivista que permita (re)pensar la lógica social del mundo contemporáneo. En la línea que plantea Grimson (2011), es ésta la pregunta teórica y política que debemos hacernos los investigadores sociales para un análisis cultural y político comprometido, que huya de determinismos y pares conceptuales, como estructura/agente, material/ideal, objetivo/subjetivo, holismo/individualismo. Estos paired concepts, según la expresión de Reinhard Bendix y Bennett Berger (1959), nos llevan a ver el mundo social de manera dicotómica, al invitarnos a elegir un frente: lo colectivo en frente de lo individual, lo subjetivo contra lo objetivo... y lo cierto es que tanto teórica, metodológica como políticamente no podemos concebir el mundo bajo esa polaridad.

En un polo encontrábamos al objetivismo, cuyas consecuencias éticas y políticas llevaron a comprender la cultura y la identidad como dos elementos indisociables. Se asumió que las diferencias culturales estaban distribuidas en el espacio, cada una con su relativa homogeneidad, con fronteras claramente definidas y una identidad más o menos propia. De esta forma, las nociones de territorio, sociedad, comunidad, cultura e identidad se encontraron totalmente anudadas entre sí. En el polo opuesto, el subjetivismo desplegó una fuerte crítica al concepto antropológico de cultura, cuestionando si en realidad existía esa correspondencia entre territorio, comunidad e identidad como afirmaba el esencialismo, lo que a la larga los llevó a cuestionar cualquier acepción al concepto de cultura.

Ante esto, el intersubjetivismo configuracional emerge como una posición posconstructivista, cuya singularidad se sintetiza en el reconocimiento de que las prácticas sociales son ontológicamente subjetivas y epistemológicamente objetivas. La relevancia de este enunciado se encuentra en la doble operación a partir de la cual se asume el postulado central del subjetivismo que expresa que la realidad es una construcción social y, al mismo tiempo, se afirman las consecuencias concretas de esa realidad en la vida de los sujetos. Esto es central ya que, si la noción de construcción social fue, quizás, el aporte más significativo de la perspectiva subjetivista para desarmar la maquinaria del pensamiento esencialista/objetivista, faltaron allí herramientas que permitan rearmar un marco interpretativo que nos permita pensar nuestra contemporaneidad. Así, mientras lo "ontológicamente subjetivo" contiene toda la potencia del reconocimiento de las subjetividades, lo "epistemológicamente objetivo" nos amplía el campo de visibilidad, abriéndonos a dominios a los cuales tanto el objetivismo como el subjetivismo fueron incapaces de abrirse: el poder, la desigualdad, la heterogeneidad, la historicidad y el conflicto.

\section{Bibliografía}

Abu-Lughod, Lila (2005): La interpretación de la (s) cultura (s) después de la televisión. Etnografías contemporáneas, l(1), pp. 57-90.

-- (2012): Escribir contra la cultura (Pilar Castro Gómez, trad). En Andamios: Revista de Investigación Social de la Universidad Autónoma de la Ciudad de México, 19(9), pp. 129-157. (Obra original publicada en 1991).

Anderson, Benedict (1991). Introducción. En Comunidades Imaginadas. México: Fondo de Cultura Económica.

Barth, Frederik (2000): A análise da cultura nas sociedades complexas. En $O$ Guru, o iniciador $e$ outras variações antropológicas. Rio de Janeiro: Contracapa. Pp. 107 -119. 
Bourdieu, Pierre (1998): Títulos y cuarteles de nobleza cultural. En La Distinción. Madrid: Taurus.

Briones, Claudia (2007): Teorías performativas de la identidad y performatividad de las teorías. En Tabula Rasa, 6, pp. 55-83.

Brubaker, Rogers y Cooper, Federick (2001): Más allá de la identidad. En Apuntes de investigación del Cecyp, 7, pp. 30-69.

Brumann, Christopher (1999): Writing for Culture: Why a successful concept should not be discarded. En Current Anthropology, 40(1), pp. 1-27.

Caggiano, Sergio (2012): El sentido común visual. Buenos Aires: Miño y Dávila.

Chakrabarty, Dipesh (2008): La provincialización de Europa en los tiempos de la globalización. En Al margen de Europa. Pensamiento poscolonial y diferencia histórica. Barcelona: Tusquets.

Canessa, Andrew (2008): El sexo y el ciudadano: Barbies y reinas de belleza en la era de Evo Morales. En P. Wade, F. Urrea Giraldo y M. Viveros Vigoya (eds.), Raza, etnicidad y sexualidades. Ciudadanía y multiculturalismo en América Latina. Bogotá: Universidad Nacional de Colombia - Centro de Estudios Sociales (CES).

García Canclini, Néstor (1999): La globalizacián imaginada, Buenos Aires-Barcelona, Paidós.

-- (2001): Culturas híbridas, Barcelona, Paidós.

Geertz, Clifford (1987): Ritual y cambio social: un ejemplo javanés. En La Interpretación de las Culturas. México: Gedisa.

Grimson, Alejandro (2010): Cultura, identidad: dos nociones distintas. En Social Identities, 16(1), pp. 63-79.

-- (2011): Los límites de la cultura. Buenos Aires: Siglo XXI.

Grimson, Alejandro, Merenson, S., y Noel, G. (2011): Introducción. En Antropología ahora. Buenos Aires: Siglo XXI.

Grüner, Eduardo (2002): El fin de las pequeñas historias. De los estudios culturales al retorno (imposible) de lo trágico. Buenos Aires: Paidós.

Hall, Stuart (1996): Introducción: ¿Quién necesita identidad? En S. Hall y P. Du Gay (coord.) Cuestiones de identidad cultural. Buenos Aires-Madrid: Amorrotu.

Hannerz, UIf (1996): Conexiones lransnacionales, Madrid, Cátedra.

Hobsbawm, Eric y Ranger, Terence (1989): The Invention of Tradition. Cambridge: Cambridge Univ. Press.

Huntington, Samuel (2004): La nueva era en la política mundial. En El choque de civilizaciones. Buenos Aires: Paidós.

-- (2004b): Who are we? The challenges to america's national identity. Nueva York: Simon\&Schuster.

Lander, Edgardo (2000): La colonialidad del saber: eurocentrismo y ciencias sociales. Perspectivas Latinoamericanas. Buenos Aires: CLACSO.

Restrepo, Eduardo y Rojas, Axel (2010): Inflexión decolonial: fuentes, conceptos y cuestionamientos. Colombia: Universidad del Cauca

Restrepo, Eduardo (2015): "Estudios culturales en América Latina". Revista de Estudos Culturais $\mathrm{N}^{\circ} 1$.

Said, Edward (2013): Orientalismo (2 ${ }^{\mathrm{a}}$ ed.). Barcelona: DeBolsillo. (Obra original publicada en 1978).

Todorov, Tveztan (1991): Nosotros y los otros. México: Siglo XXI.

Viveiros de Castro, Eduardo (1999): Etnología Brasileira. En S. Miceli (org) O que ler na ciencia social brasileira (1970-1995), Antropología (Volume I), Sao Paulo Editora Sumaré: ANPOCS-Brasilia DF:CAPES.

Williams, Raymond (1980): Marxismo y literatura. Barcelona: Península. 